\title{
Computations of Compressible Multifluids
}

\author{
Rémi Abgrall* and Smadar Karni ${ }^{*} 1$ \\ * Mathématiques Appliquées, University of Bordeaux, 351 Cours de la Libération, \\ 33405 Talence Cedex, France; and $\dagger$ Department of Mathematics, \\ University of Michigan, Ann Arbor, Michigan 48109-1109 \\ E-mail: Karni@math.lsa.umich.edu
}

Received December 16, 1999; revised November 17, 2000

\begin{abstract}
Recent years have seen a growing interest in developing numerical algorithms for compressible multifluids. Computations ran into unexpected difficulties due to oscillations generated at material interfaces, and understanding of the underlying mechanisms was needed before these oscillations could be circumvented. This paper reviews some of the recent models and numerical algorithms that have been proposed and points to key ideas that they have in common. Noting the known fact that such oscillations do not arise in single-fluid computations, an extremely simple algorithm is proposed which circumvents the oscillations and amounts to computing two different flux functions across material fronts, to update the different fluids on both sides. (c) 2001 Academic Press
\end{abstract}

\section{INTRODUCTION}

Recent years have witnessed a growing interest in developing numerical methods suitable for computing multimaterial flows and in their efficient implementation to studying complex flow phenomena $[1-3,7,9,11-14,16-24,26,31-37,39]$. Understanding the dynamics of fluids consisting of several fluid components is of great interest in a wide range of physical flows, including studying the dynamics and stability of bubbles and interfaces, mixing processes, bubbly flows, and liquid suspensions. Such flows give rise to challenging problems, both theoretically and computationally. In this paper, we focus on models and numerical methods for multimaterial flows consisting of pure fluids separated by material interfaces.

\footnotetext{
${ }^{1}$ Work supported in part by ONR Award N000149910449 and NSF award DMS 9973291. This work was carried out in part while the author visited the University of Bordeaux.
} 
Assuming all fluid components can be described by a single velocity and a single pressure function, the flow is described by the compressible Euler equations

$$
\left(\begin{array}{c}
\rho \\
\rho u \\
E
\end{array}\right)_{t}+\left(\begin{array}{c}
\rho u \\
\rho u^{2}+p \\
u(E+p)
\end{array}\right)_{x}=0, \quad p(\rho, e)
$$

expressing conservation of mass, momentum, and energy of the fluid mixture, and its thermodynamics properties given by the equation of state (EOS). Here we use $\rho, u$, and $p$ to denote density, velocity, and pressure; $E=\rho e+\frac{1}{2} \rho u^{2}$ denotes the total energy; and $e$ is the specific internal energy. We further use $a$ to denote the speed of sound and $H=(E+p) / \rho$ to denote the specific total enthalpy. The flow description is completed by providing an additional equation that describes the dynamics of the fluid composition, and by specifying its effective thermodynamics, i.e., the EOS of the mixture.

For the rest of the paper, we assume that the fluid consists of two components $\Sigma_{1}$ and $\Sigma_{2}$. The EOS depends on the fluid composition, described by the variable $\phi$. Various choices of $\phi$ have been suggested in the literature, depending on the model assumptions. For example, in [30] it was suggested to take $\phi$ to be the specific heat ratio of the fluid $\gamma$, in $[1,2,4,23]$, the mass fraction was used, and in $[12,26]$, the level-set function (see Section 2) was used. For all these models, the governing equation for the variable $\phi$ has the form

$$
(\rho \phi)_{t}+(\rho u \phi)_{x}=0
$$

The EOS for ideal fluids is

$$
\rho e=\frac{\rho}{\gamma-1},
$$

where $\gamma=\gamma(\phi)$ denotes the effective ratio of specific heats of the fluid mixture. A more general EOS for stiff fluids is

$$
\rho e=\frac{p+\gamma p_{\infty}}{\gamma-1}
$$

where $p_{\infty}=p_{\infty}(\phi)$ is a stiffness parameter, and the choice $p_{\infty}=0$ recovers the ideal gas case. We shall focus on these two cases in the paper. They can be seen as simplifications of more complex fluids of the Mie-Grüneisen type

$$
p=p_{\infty}(\phi, \rho)+\gamma(\phi)\left(\rho e-e_{\infty}(\phi, \rho)\right)
$$

for which the numerical phenomena we describe in the paper become even more severe.

Using $W=(\rho, \rho u, E, \rho \phi)^{T}$ and $F(W)=\left(\rho u, \rho u^{2}+p, u(E+p), \rho u \phi\right)^{T}$ to denote the vector and dependent variables and flux functions, the equations take the form

$$
W_{t}+F(W)_{x}=0
$$

A choice needs to be made as to the general computational framework for problems involving interface dynamics: (i) Front-tracking methods treat discontinuities as moving internal boundaries, and the jump conditions are imposed (see for example [5] and references therein). Such methods are attractive in that interfaces are preserved as sharp discontinuities, 
but they are difficult to implement in several space dimensions. (ii) Front-capturing methods are derived from the integral form of the governing equations. They are straightforward to implement in any number of space dimensions but come at a price of smearing solution discontinuities. The methods reviewed in this paper are shock-capturing methods, with a certain tracking "flavor" dictated by the nature of flows dominated by propagating interfaces.

The genral framework for shock-capturing algorithms considered in this paper is that of Godunov-type discretization [15]. A numerical grid is given by the mesh spacing $\Delta x$ and time step $\Delta t, x_{j}=j \Delta x$, and $t_{n}=n \Delta t$. Intergrating (4) over the box $\left[x_{j-\frac{1}{2}}, x_{j+\frac{1}{2}}\right] \times$ $\left[t_{n}, t_{n+1}\right]$ gives

$$
W_{j}^{n+1}=W_{j}^{n}-\frac{\Delta t}{\Delta x}\left(F_{j+\frac{1}{2}}^{n+\frac{1}{2}}-F_{j-\frac{1}{2}}^{n+\frac{1}{2}}\right)
$$

where

$$
W_{j}^{n}=\frac{1}{\Delta x} \int_{x_{j-\frac{1}{2}}}^{x_{j+\frac{1}{2}}} W\left(x, t_{n}\right) d x, \quad F_{j \pm \frac{1}{2}}^{n+\frac{1}{2}}=\frac{1}{\Delta t} \int_{t_{n}}^{t_{n}+1} F\left(x_{j \pm \frac{1}{2}}, t\right) d t
$$

are the cell-average of the solution over grid-cell $j$ and the time-average flux functions at cell interfaces respectively.

The paper is organized as follows. In Section 2 we describe the general structure of the multifluid flow model (Eqs. (1)-(3)) and present several specific models. In Section 3 we describe the numerical difficulties that arise in discretizing such models and that result in oscillations and other computational inaccuracies near material fronts. We then describe several approaches for overcoming those difficulties within the general framework of the model proposed, as well as other approaches that do not fall exactly in this framework. In particular, we point out the fact that these difficulties do not arise in computations involving single fluids and that some recent successful multifluid algorithms capitalize precisely on this property. In Section 4 we then propose an extremely simple algorithm with a singlefluid flavor. It circumvents the difficulties by computing two different flux functions across material fronts to update the different fluids on both sides. The conservation properties of the algorithm are analyzed. In Section 5, we present a variety of numerical examples involving shock-interface interaction for ideal and stiff fluids as well as flows with chemical reaction. Conservation errors are studied numerically, and convergence is demonstrated. We end up with concluding remarks in Section 6.

\section{MULTIFLUID MODELS}

\subsection{General Structure}

The extended system (1)-(3) remains time-hyperbolic. We set $\epsilon=\rho e$ and denote $\kappa=$ $\gamma-1=p_{\epsilon}$. The right eigenvector matrix and associated eigenvalues for EOS (3b) are given by

$$
R=\left(\begin{array}{cccc}
1 & 1 & 0 & 1 \\
u-a & u & 0 & u+a \\
H-u a & \frac{u^{2}}{2} & -p_{\phi} & H+u a \\
\phi & \phi & p_{\epsilon} & \phi
\end{array}\right)
$$


and

$$
\Lambda=\operatorname{diag}(u-a, u, u, u+a)
$$

where the speed of sound satisfies

$$
a^{2}=(\gamma-1)\left(H-\frac{1}{2} u^{2}\right)=\gamma \frac{p+p_{\infty}}{\rho} .
$$

The particle field becomes degenerate with a double eigenvalue corresponding to the contact surface being not merely an entropy wave front but also a material interface.

The jump conditions $[F(W)]=\sigma[W]$ and Riemann invariants can be summarized as follows:

- Material interfaces: $[u]=0$; hence $[p]=0$ and $\sigma=u$. The jumps in $\phi$ and $\rho$ are arbitrary. A material interface is the only location where the composition of the fluid can change.

- Shock waves: $[u] \neq 0$; hence $[p] \neq 0$. Here, $[\phi]=0$, so that the classical shock jump conditions apply [10].

- Across a $(u \mp a)$ simple wave, the Riemann invariants are

$$
\phi, \quad\left(p+p_{\infty}\right) \rho^{-\gamma}, \quad u \pm \frac{2}{\gamma-1} a
$$

\subsection{Some Examples}

We now describe some specific models that have been proposed and discussed extensively in the literature.

\subsection{1. $\gamma$-Model}

In [31], $\gamma$ is extended to be a piecewise constant function $\gamma(x, t)$. Being a state variable, $\gamma$ propagates with the fluid velocity, $u$, and hence satisfies

$$
\gamma_{t}+u \gamma_{x}=0
$$

which for convenience can be combined with (1a) and cast in conservation form

$$
(\rho \gamma)_{t}+(\rho u \gamma)_{x}=0
$$

The numerically computed $\gamma$ consists of piecewise constant values connected by transition layers, interpreted as "numerical mixing" of the species. We note the following:

- The local value of $\gamma$, computed from $\gamma=(\rho \gamma) / \rho$, is not guaranteed to lie in the physically acceptable range (between $\gamma_{1}$ and $\gamma_{2}$ ), and overshoots may and do occur (see analysis in Section 3.1; also see [6]).

- Any function $f(\gamma)$ is also a state variable; hence (6) can be replaced by

$$
f(\gamma)_{t}+u f(\gamma)_{x}=0
$$


It turns out that the particular choice of $f(\gamma)=1 / \gamma-1$, with appropriate discretization, offers clear advantages. This is further discussed in Section 3.2.2 (see also [2]).

A Roe-type linerization was proposed [31] for the extended model (discussed also in the more accessible reference [19]). Numerical computations with this model suffer inaccuracies near the material fronts. The velocity profile shows a persistent nonphysical jump across isolated material fronts [19]. (See Fig. 3a and further discussion in Section 5.)

\subsubsection{Mass Fraction Model}

In $[1,23,24]$ the mass fraction $Y_{1}=\rho_{1} /\left(\rho_{1}+\rho_{2}\right)=\rho_{1} / \rho$ (respectively $\left.Y_{2}\right)$ is used to describe the concentration of $\Sigma_{1}$ (respectively $\Sigma_{2}$ ) in the fluid mixture. The mass conservation equation for species $\Sigma_{1}$ is given by

$$
(\rho Y)_{t}+(\rho u Y)_{x}=0
$$

where $Y \equiv Y_{1}=1-Y_{2}$. Using $p_{1,2}$ to denote the partial pressures of species $\Sigma_{1,2}$, and assuming that the species are calorically perfect gases in thermal equilibrium with specific heat constants $C_{v}$ and $C_{p}$, one has

$$
\begin{array}{ll}
e_{1}=C_{v 1} T, & p_{1}=\left(\gamma_{1}-1\right) \rho_{1} e_{1}, \\
e_{2}=C_{v 2} T, & p_{2}=\left(\gamma_{2}-1\right) \rho_{2} e_{2} .
\end{array}
$$

Using Dalton's law of partial pressures, $p=p_{1}+p_{2}$, yields $\rho e=\rho_{1} e_{1}+\rho_{2} e_{2}=\rho C_{v} T$, where $C_{v}(Y)=Y_{1} C_{v 1}+Y_{2} C_{v 2}$ and

$$
p=(\gamma-1) \rho e, \quad \text { where } \gamma=\gamma(Y)=\frac{Y_{1} C_{p 1}+Y_{2} C_{p 2}}{Y_{1} C_{v 1}+Y_{2} C_{v 2}} .
$$

The computational difficulties in this model are similar to those observed with the $\gamma$-model:

(i) the mass fraction $Y$, computed from $(\rho Y) / \rho$, is not guaranteed to remain in the physically relevant range of $[0,1]$.

(ii) The velocity profiles show a persistent nonphysical jump across the material front (see Fig. 3b). The first of these observations was analyzed in [23] and is attributed to the fact that $\rho$ and $\rho Y$ are not varying "in-phase" with each other across material fronts. (See Section 3.1.)

Removing the assumption of thermal equilibrium leads to an effective $\gamma$ for the mixture [36] of

$$
\gamma(Y)=\frac{\gamma_{1} Y_{1} e_{1}+\gamma_{2} Y_{2} e_{2}}{Y_{1} e_{1}+Y_{2} e_{2}},
$$

but it requires in addition to (1)-(3) that one also solves for the internal energies of the individual species.

\subsubsection{Level-Set Model}

An "immiscible" model was proposed in [26] where a level-set function $\psi$ is used to track the material interface. The fluid "mixture" consists of either fluid $\Sigma_{1}$ or fluid $\Sigma_{2}$ and 
thermodynamic properties change discontinuously across the material interface. A marker variable $\psi$ is initialized so that its zero level-set defines the material interface at $t=0$, and it is positive (respectively negative) in the domain occupied by fluid component $\Sigma_{1}$ (respectively $\Sigma_{2}$ ). Then, $\psi$ satisfies a transport equation similar to (6), which again may be combined with (1a) and recast in conservation form

$$
(\rho \psi)_{t}+(\rho u \psi)_{x}=0
$$

By definition, $\psi(x, t)=0$ at the interface at all later times $t>0$, and the EOS is given by (3a) with

$$
\gamma(\psi)= \begin{cases}\gamma_{1} & \psi>0 \\ \gamma_{2} & \psi<0\end{cases}
$$

Computations with this model, using a Roe-type upwind scheme, suffer from even more pronounced oscillations near material fronts [20] (see Fig. 3c).

As discussed in Section 3.2, the above models produce spurious pressure oscillations across material fronts. Analysis of the above models presented in [19] shows that the pressure oscillations in the $\gamma$-model and mass-fraction model are $\mathcal{O}(\Delta \gamma)^{2}$ and are of a definite sign, while in the level-set model, they are of order $\mathcal{O}([\gamma])$ and do not have a definite sign. This is consistent with the form of the computational inaccuracies observed in Fig. 3.

\section{WHAT IS GOING WRONG?}

\subsection{Positivity of Mass Fractions}

The following discussion focuses on the mass-fraction model but applies equally to the $\gamma$-model. While physically $Y \in[0,1]$, the numerically computed value of $Y,(\rho Y) / \rho$, is not guaranteed to lie in this range. As discussed below, this problem has been observed with the best available schemes and is referred to as the "positivity of the mass fraction" problem.

We first note that the occurrence of negative mass fraction is not a result of a timestepping stability violation. Indeed, some schemes may develop negative mass fractions for any time step, however small. As shown in [4], applying Roe's scheme to the shock tube data in Table I leads to a negative $Y$ after one time step, for any choice of time step $\Delta t>0$. An example with Osher's solver is also given in [4]. Note that here $\gamma_{L}=\gamma_{R}$, establishing that the positivity problem is unrelated to the presence of multispecies as is often believed.

TABLE I

Shock-Tube Data That Produce Negative Mass Fraction

\begin{tabular}{lrrrrr}
\hline & $\rho$ & $u$ & $H$ & $Y$ & $\gamma$ \\
\hline Left & 1 & -1 & 1 & 1 & 1.4 \\
Right & 1 & 1 & 5 & 0 & 1.4 \\
\hline
\end{tabular}


To cure this problem, a scheme property that ensures positivity of the mass fractions has been identified in [23], that the flow rate of $\rho Y$, namely $F_{\rho Y}$, is varying "in phase" with the flow rate of the entire fluid mixture $\rho$, namely $F_{\rho}$. To that end, it is proposed to modify the species mass flux as

$$
F_{\rho Y}^{\text {modified }}=Y^{*} F_{\rho}
$$

with

$$
Y^{*}= \begin{cases}Y_{L} & \text { if } F_{\rho}>0, \\ Y_{R} & \text { if } F_{\rho}<0,\end{cases}
$$

with $L, R$ denoting the states neighboring the cell-interface where $F$ is being evaluated.

Any scheme that satisfies this condition, for example the Godunov scheme [15] and van Leer flux splitting scheme [38], preserves the positivity of the mass fractions, provided the CFL condition is satisfied.

\subsection{Pressure Oscillations}

A major difficulty in discretizing the multifluid Eqs. (1)-(3) is ensuring that pressure equilibrium between fluid components is not disturbed by the numerical scheme. Indeed, the best available schemes develop pressure oscillations near material fronts separating fluids in pressure equilibrium. These oscillations are not the ones commonly associated with high-order numerical methods. They are present already in first-order computations (see Fig. 3) and are not removed by going to a higher order. We note that such oscillations are not observed in the single-fluid model, and as we discuss below, a number of recent schemes capitalize precisely on this property. To shed light on the multifluid case, a brief analysis of the evolution of the pressure field is carried out, first for a single fluid, and then for a multifluid mixture.

Using $\delta()=()^{n+1}-()^{n}$ to denote time changes, $\Delta()=()_{j+\frac{1}{2}}-()_{j-\frac{1}{2}}$ spatial variations, and $v=\frac{\Delta t}{\Delta x}$ the mesh ratio, the Godunov scheme applied to (4) gives

$$
\left(\begin{array}{c}
\rho \\
\rho u \\
E
\end{array}\right)_{j}^{n+1}=\left(\begin{array}{c}
\rho \\
\rho u \\
E
\end{array}\right)_{j}^{n}+\left(\begin{array}{c}
\delta \rho \\
\delta(\rho u) \\
\delta E
\end{array}\right)
$$

where

$$
\left(\begin{array}{c}
\delta \rho \\
\delta(\rho u) \\
\delta E
\end{array}\right)=-v\left(\begin{array}{c}
\Delta(\rho u) \\
\Delta\left(\rho u^{2}+p\right) \\
\Delta(u(E+p))
\end{array}\right)
$$

\subsubsection{Single Fluid}

In the single- (ideal) fluid case, $\gamma_{j}^{n}=\gamma$ is uniform, and the new pressure $p_{j}^{n+1}=p+\delta p$ is obtained using the EOS (3a). Dropping the super- and subscripts for notational convenience we have

$$
\frac{p+\delta p}{\gamma-1}=(E+\delta E)-\frac{1}{2}(\rho+\delta \rho)(u+\delta u)^{2} .
$$


Across isolated material fronts, the exact solution has uniform $u$ and $p$. Numerically, if $u$ and $p$ are uniform in the data, $u_{j}^{n}=u_{j \pm \frac{1}{2}}=u, p_{j}^{n}=p_{j \pm \frac{1}{2}}=p$, and the solution updates (11) reduce to

$$
\begin{aligned}
& \delta \rho=-v u \Delta \rho, \\
& \delta(\rho u)=-v u^{2} \Delta \rho=u \delta \rho, \\
& \delta E=-v u \Delta E=-v \frac{1}{2} u^{3} \Delta \rho \\
& \quad=\frac{1}{2} u^{2} \delta \rho .
\end{aligned}
$$

From (13a,b), it readily follows that

$$
\delta u=0
$$

and from (13c) and (12) it follows that

$$
\delta p=0,
$$

implying that the computed $u_{j}^{n+1}$ and $p_{j}^{n+1}$ remain uniform, as they should.

\subsubsection{Multifluids}

In the multifluid case, $\gamma=\gamma_{j}^{n}$ is generally not uniform and varies across the material front. The new pressure then becomes

$$
(p+\delta p)=(\gamma+\delta \gamma-1)\left((E+\delta E)-\frac{1}{2}(\rho+\delta \rho)(u+\delta u)^{2}\right) .
$$

Fully conservative methods. By fully conservative methods, we mean methods that not only conserve total mass, momentum, and energy of the fluid mixture but also conserve mass of the individual species. We therefore refer here only to the discretizations of the mass-fraction model, the only model with this property.

In the mass-fraction model, $\gamma=\gamma(Y)$, and

$$
\gamma_{j}^{n+1}=\gamma\left(Y_{j}^{n+1}\right)=\gamma+\delta \gamma
$$

Consider a flow consisting of a material front separating two ideal gases with $\gamma_{l, r}$, moving with some positive velocity $u>0$, with initial data

$$
W_{J}^{0}= \begin{cases}\left(\rho, \rho u, \frac{p}{\gamma_{l}-1}+\frac{\rho}{2} u^{2}, \rho\right) & j<1 \\ \left(\rho, \rho u, \frac{p}{\gamma_{r}-1}+\frac{\rho}{2} u^{2}, 0\right) & j \geq 1\end{cases}
$$

In this case, the numerical flux reduces to the upwind flux,

$$
F_{j+1 / 2}=F\left(W_{j}\right)
$$

and after one time step, we have

$$
W_{1}^{1}=W_{1}^{0}-v\left(F\left(W_{1}^{0}\right)-F\left(W_{0}^{0}\right)\right) .
$$


Some simple algebra shows that after one time step

$$
\begin{aligned}
& Y_{1}^{1}=1-v u, \\
& u_{1}^{1}=u,
\end{aligned}
$$

which is the correct solution behavior. However, the pressure computation gives

$$
\frac{p_{1}^{1}}{\gamma_{1}^{1}-1}=\frac{p}{\gamma_{l}-1}-v u p\left(\frac{1}{\gamma_{l}-1}-\frac{1}{\gamma_{r}-1}\right) .
$$

Using (8) we then get

$$
\gamma_{1}^{1}=\gamma\left(Y_{1}^{1}\right)=\frac{C_{p 1}-v u\left(C_{p 1}-C_{p 2}\right)}{C_{v 1}-v u\left(C_{v 1}-C_{v 2}\right)} .
$$

If $0<v u<1$,

$$
\gamma_{l}<\gamma_{1}^{1}<\gamma_{r}
$$

in which case the newly computed pressure $p_{1}^{1} \neq p$, and a pressure oscillation is generated. The oscillation is $\mathcal{O}(\Delta \gamma)^{2}$ and has a definite sign (see [19]).

This result is quite general. It is shown in [1] that any Godunov-type scheme which is fully conservative will not be able to maintain pressure equilibrium and will develop a pressure oscillation across material fronts. This suggests that one may need to move away from the strictly conservative framework. An early paper to adopt a fully nonconservative approach for multifluids was [19]. Subsequently, several nonstrictly conservative schemes were proposed [2, 12, 18, 20, 34, 36, 37]. We discuss some of them in Section 3.3.

Nonconservative methods. Again, considering isolated material fronts, we denote $\alpha=$ $\frac{1}{\gamma-1}$. Equation (16) now becomes

$$
(p+\delta p)(\alpha+\delta \alpha)=(E+\delta E)-\frac{1}{2}(\rho+\delta \rho)(u+\delta u)^{2}
$$

with

$$
\begin{aligned}
\delta E & =-v u \Delta E \\
& =-v u \Delta\left(\frac{p}{\gamma-1}-\frac{1}{2} \rho u^{2}\right) \\
& =-\frac{1}{2} u^{2} \delta \rho-v u p \Delta\left(\frac{1}{\gamma-1}\right) .
\end{aligned}
$$

Inserting (18) into (17) we now get

$$
\begin{aligned}
(p+\delta p)(\alpha+\delta \alpha) & =E+\delta E-\frac{1}{2}\left(\rho+\delta \rho(u+\delta u)^{2}\right. \\
& =E-\frac{1}{2} \rho u^{2}-\nu u p \Delta\left(\frac{1}{\gamma-1}\right) \\
& =p \alpha-v u p \Delta \alpha,
\end{aligned}
$$

where we have used the fact that, as in the single-fluid case, $\delta u=0$. 
It is now clear that in order for $\delta p$ to vanish, we must have

$$
\alpha+\delta \alpha=\alpha-v u \Delta \alpha
$$

This was realized in [2] and was later extended to multifluids with stiff EOS [32, 34], to Van der Waals EOS [35], and to multiphase flows [33]. Clearly if condition (19) is not satisfied, then even idealized situations such as isolated material fronts will produce pressure oscillations.

Finally, we note that (19) is a discretization of the transport equation

$$
\alpha_{t}+u \alpha_{x}=0
$$

Referring back to Eq. (7), we see that any function $f(\gamma)$ is a state function and is effectively transported by the fluid. The simple analysis carried out above shows that the one choice of $f(\gamma)$ that does not produce pressure oscillations is $f(\gamma)=\frac{1}{\gamma-1}$, provided it is discretized by (19).

The above algebra is general and applies to any scheme that preserves uniform velocity across material fronts [2]. This condition is not trivially satisfied. We note that Godunov-type schemes fulfill this condition, but flux-splitting schemes, such as van Leer's [38] (see [2] for details) do not.

Condition (19) was obtained under the ideal assumption that the flow consists of an isolated material front. It implies that in order for $\delta p=0$, Eq. (20) cannot be discretized independently of the discretization of the conserved variables in (1). More generally, although $u$ and $p$ are continuous across a contact surface, they are not necessarily uniform. Requiring that $\delta p=0$ is no longer valid, and Eq. (19) needs to be generalized.

The generalization follows the same guiding principle: Combining the evolution equations of the total energy, the momentum, and mass, we can obtain an evolution equation for the internal energy,

$$
\frac{\partial \rho e}{\partial t}+u \frac{\partial \rho e}{\partial x}+p \frac{\partial u}{\partial x}=0
$$

which, for ideal gases, becomes

$$
\frac{\partial \alpha p}{\partial t}+u \frac{\partial \alpha p}{\partial x}+p \frac{\partial u}{\partial x}=0
$$

or

$$
p\left(\frac{\partial \alpha}{\partial t}+u \frac{\partial \alpha}{\partial x}\right)+\alpha\left(\frac{\partial p}{\partial t}+u \frac{\partial p}{\partial x}+\rho a^{2} \frac{\partial u}{\partial x}\right)=0
$$

In the above equation, we identify the evolution equation for $\alpha$ and the pressure evolution equation. The guiding principle is to discretize (20) in such a way that the resulting changes in $p$, namely $\delta p$, are consistent with changes implied by the pressure evolution equation (see [2] for details). Finally, this construction can be generalized to high order [2] and to unstructured meshes [3]. 
Schemes that are discretized in this fashion conserve mass, momentum, and total energy of the fluid mixture (but not mass of the individual species), they admit a maximum principle on $\alpha$ under a CFL-like condition, and they preserve the positivity of mass fractions. A second-order extension of the above method, derived in detail in [2], was implemented in the numerical section methods and is referred to as the "quasi conservative scheme." It clearly prevents oscillations in the pressure.

\subsection{Other Approaches}

\subsubsection{Volume-of-Fluid Model}

The volume-of-fluid method goes back to an early paper by Noh and Woodward [27]. It was formalized in a mathematical framework in an unpublished paper by Colella, Glaz, and Ferguson (CGF) in [8], which also accounts for the different compressibilities of the species. A discussion of the CGF method appeared in [25], where it is also extended to condensed media problems. The CGF volume-of-fluid model is essentially equivalent to a multiphase fluid model with a single pressure and a single velocity and is not of the general form (1-3). In this formulation, the balance equations for mass and energy are written for the individual species, while the momentum equations are written for the fluid mixture. The usual mass and energy conservation for the fluid mixture are obtained upon summation of the species equations.

Evolution equations are derived for the species volume fractions, $f_{\alpha}$,

$$
\frac{\partial f_{\alpha}}{\partial t}+\frac{\partial u f_{\alpha}}{\partial x}=f_{\alpha} \frac{\hat{\Gamma}}{\gamma_{\alpha}} u_{x}
$$

assuming that compressions are isentropic, and requiring that changes in $f_{\alpha}$ all correspond to the same pressure change. The latter ensures that most volume changes are absorbed by the most compressible fluid. These requirements lead to two effective $\gamma$ 's for the fluid mixture: (i) $\hat{\gamma}$ to be used in the computation of the internal energy and (ii) $\hat{\Gamma}$ to be used in the computation of the speed of sound,

$$
\frac{1}{\hat{\gamma}-1}=\frac{f_{1}}{\gamma_{1}-1}+\frac{f_{2}}{\gamma_{2}-1}, \quad \frac{1}{\hat{\Gamma}}=\frac{f_{1}}{\gamma_{1}}+\frac{f_{2}}{\gamma_{2}} .
$$

The algorithm evolves the fluid mixture as a single fluid. The volume fractions and the species thermodynamics are used to compute effective thermodynamic quantities for the fluid mixture. The changes in the mixture mass and total energy, given by the flux difference, are then distributed among the fluid component. The species EOSs are then used to compute the species pressure and internal energy. While the algorithm preserves pressure equilibrium between the multifluid components, near shocks, different species may develop different pressures and a further relaxation process is required to restore pressure equilibrium. Assuming isentropic compression, the equilibrium pressure is given by

$$
\frac{\hat{p}}{\hat{\gamma}}=\frac{p_{1} f_{1}}{\gamma_{1}}+\frac{p_{2} f_{2}}{\gamma_{2}} .
$$


The resulting changes in volume fractions are computed, and the total energy of the individual species is recomputed to account for the work done by the pressure forces due to pressure relaxation. See [25] for more details.

\subsubsection{Pressure Evolution Model}

The evolution equation for the pressure is

$$
p_{t}+u p_{x}+\rho a^{2} u_{x}=0 .
$$

It provides a valid flow description for smooth flows as well as near contact surfaces, where changes in pressure or velocity have finite amplitude. In particular, if both $u$ and $p$ are uniform in the data, $p$ remains uniform in the solution, and any consistent discretization of (22) automatically inherits this property. Near shocks, however, (22) is not valid, and conservation errors will occur, translating into incorrect shock strength or speed.

In [19], a nonconservative model based on the primitive variables $U=(\rho, u, p)$ was presented. This model uses small viscous correction terms to remove leading-order conservation errors. The correction terms are obtained by transforming the governing equations from conservative to primitive formulation together with the small $(O(h))$ numerical viscosity terms. Being based on the primitive variables, the model lends itself to oscillation-free discretizations. Conservation errors, however, are eliminated only to leading order, which limits the suitability of the method to shocks of weak to moderate strength $\left(M_{s} \approx 2.5\right)$, where conservation errors are within less than $2 \%$. The method was successfully applied to study a variety of shock-interface interaction problems [19] and was successfully applied to study the highly complex interaction of weak shock waves with bubbles [28].

This approach was modified in [20] to suit also strong shocks (up to $M_{s} \approx 100$ ). The idea is to use (22) only across material fronts but otherwise to revert back to the conservative formulation (1). For the level-set formulation, the following hybrid model is proposed [20]. Solve

$$
\left(\begin{array}{c}
\rho \\
\rho u \\
\rho \psi
\end{array}\right)_{t}+\left(\begin{array}{c}
\rho u \\
\rho u^{2}+p \\
\rho u \psi
\end{array}\right)_{x}=0
$$

together with one of the following:

(i) If $\psi=0$ (material front) then solve

$$
p_{t}+u p_{x}+\rho a^{2} u_{x}=0 \Longrightarrow E=\frac{p}{\gamma(\psi)-1}+\frac{1}{2} u^{2}
$$

(ii) otherwise (away from material front)

$$
E_{t}+(u(E+p))_{x}=0 \Longrightarrow p=(\gamma(\psi)-1)\left(E-\frac{1}{2} u^{2}\right) .
$$

This model leads to essentially conservative algorithms in that conservation error occurs only near the material front. The error is only in the total energy; it is extremely small even for very strong chocks $(\approx 0.2 \%)$ and converges to zero with mesh refinement [20]. 
This approach was applied to compute shock-interface interaction of ideal gases as well as stiff fluids and interfacial instabilities in multifluid dynamics and to bubble dynamics with surface tension [20-22].

\subsubsection{Internal Energy Correction Algorithm}

The discretization of the energy equation has the general form

$$
\begin{aligned}
E_{j}^{n+1} & =\left(\frac{p}{\gamma-1}\right)_{j}^{n+1}+\left(\frac{1}{2} \rho u^{2}\right)_{j}^{n+1} \\
& =\left(\frac{p}{\gamma-1}\right)_{j}^{n}+\left(\frac{1}{2} \rho u^{2}\right)_{j}^{n}-\frac{\Delta t}{\Delta x}\left\{\Delta\left(\frac{u p}{\gamma-1}\right)_{j}^{n}+\Delta\left(\frac{1}{2} \rho u^{3}+u p\right)_{j}^{n}\right\}
\end{aligned}
$$

where we have specialized to ideal gases $\rho e=p /(\gamma-1)$.

At the heart of the algorithm proposed in [18] is a modification of the internal energies in the above algorithm,

$$
\begin{gathered}
\left(\frac{p}{\gamma-1}\right)_{j \pm \frac{1}{2}}^{n} \Rightarrow \frac{p_{j+\frac{1}{2}}^{n}}{\gamma_{j}^{n+1}-1}, \\
\left(\frac{p}{\gamma-1}\right)_{j}^{n} \Rightarrow \frac{p_{j}^{n}}{\gamma_{j}^{n+1}-1},
\end{gathered}
$$

where $\gamma_{j}^{n+1}=\gamma\left(Y_{j}^{n+1}\right)$ is given by (8). This modification effectively renders the energy update (23) a single-fluid algorithm, in that the only fluid "seen" by the algorithm, both in the data $\left(t_{n}\right)$ and in the solution $\left(t_{n+1}\right)$, is a single ideal fluid with $\gamma=\gamma_{j}^{n+1}$. Since for a single fluid, contact surfaces are preserved (see (14)-(15)), this step effectively eliminates the spurious pressure oscillations.

The internal energy correction step (24) affects the total energy balance, and the algorithm does not in general conserve the total energy. Conservation errors are confined to the vicinity of material fronts. It is also shown that the errors vanish if the fluid components are in thermal equilibrium [18].

\subsubsection{The Ghost Fluid Method}

The Ghost Fluid Method [12] is a level-set-based algorithm, which treats the interface as a moving internal boundary. Consider a material interface with fluid $A$, say, on the right. While the real cells on the left side of the interface are occupied by fluid $B$, ghost cells are introduced which are assumed to be occupied by fluid $A$. Flow variables are extended into the ghost cells. It is recommended that the extrapolated variables be $p, u$, and the entropy $s$ (see Fig. 1):

$$
\begin{array}{ll}
p_{j}^{A}=p_{j}^{B}, & p_{j+1}^{B}=p_{j+1}^{A}, \\
u_{j}^{A}=u_{j}^{B}, & u_{j+1}^{B}=u_{j+1}^{A}, \\
s_{j}^{A}=s_{j+1}^{A}, & s_{j+1}^{B}=s_{j}^{B} .
\end{array}
$$




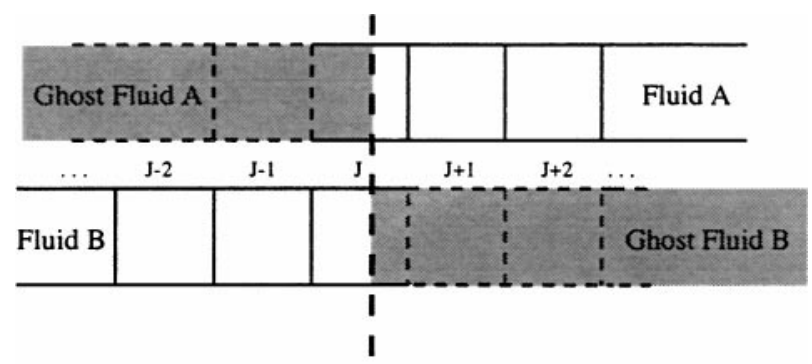

Interface

FIG. 1. Schematic for the Ghost Fluid Method [12].

The two copies of the solutions are evolved to obtain $\left(W^{A, B}\right)_{j}^{n+1}$. The level-set function $\psi$ then identifies which parts of the two copies of the solution correspond to the real fluids, and those parts are "stitched" together, for example,

$$
\rho_{j}^{n+1}= \begin{cases}\left(\rho^{A}\right)_{j}^{n+1} & \psi_{j}^{n+1}<0, \\ \left(\rho^{B}\right)_{j}^{n+1} & \psi_{j}^{n+1}>0 .\end{cases}
$$

Resulting solutions tend to be discontinuous across the material front [12]. It is rather tempting to attribute the elimination of pressure oscillations to the elimination of dissipation errors across material fronts. Yet, what eliminates the oscillations is the fact that the ghost fluid on "the other side" of the interface is thermodynamically similar, i.e., obeys the same EOS. This step essentially renders the model a single-fluid model. The oscillations do not appear because, in a single fluid, contact surfaces are respected $(14,15)$. We note that the near elimination of dissipation errors offers an advantage for problems where the EOS has a limited range of validity, as is the case with Tait's EOS [10, 12].

The extrapolation into the ghost cells (25) affects conservation of all variables, but mesh refinement tests indicate convergence [12]. We point out that populating the ghost cells by a thermodynamically similar "ghost fluid" can be done at a smaller cost in terms of loss of conservation. It can be achieved by sacrificing energy conservation only, while still conserving total mass and momentum. This point is illustrated further in the following section.

On the technical side, note that the "ghost" copy of the solution is not needed everywhere in the flow field, just in the vicinity of the material front. Keeping a narrow strip of three to five ghost cells near the interface is rather simple in 1D but is more complicated in multidimensions where the interface may become very distorted.

\section{A SIMPLE SINGLE-FLUID ALGORITHM}

Assume that two adjacent grid cells have two different fluids with states $W_{j}=W_{L}=$ $\left(\rho_{l}, u_{l}, p_{l}\right)^{T}$ with $\gamma=\gamma_{l}$ and $W_{j+1}=W_{R}=\left(\rho_{r}, u_{r}, p_{r}\right)^{T}$ with $\gamma=\gamma_{r}$. The interface flux depends on the left and right states

$$
F_{j+\frac{1}{2}}\left(W_{L}, W_{R} ; \gamma_{l}, \gamma_{r}\right)
$$




$$
F_{j+\frac{L}{2}}^{L} \neq F_{j+\frac{L}{2}}^{R}
$$

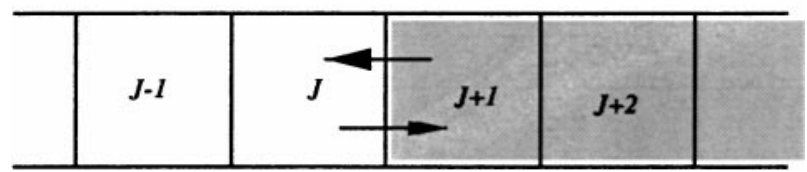

FIG. 2. Schematic for the simple single-fluid algorithm.

We propose to compute two intercell fluxes, one assuming that the fluids in both cells $j$ and $j+1$ are $\gamma_{l}$-fluids, and one assuming that both fluids are $\gamma_{r}$-fluids:

$$
\begin{aligned}
& F_{j+\frac{1}{2}}^{L}=F_{j+\frac{1}{2}}\left(W_{L}, W_{R} ; \gamma_{l}\right), \\
& F_{j+\frac{1}{2}}^{R}=F_{j+\frac{1}{2}}\left(W_{L}, W_{R} ; \gamma_{r}\right) .
\end{aligned}
$$

We then use $F_{j+\frac{1}{2}}^{L}$ to update the fluid in cell $j$ and $F_{j+\frac{1}{2}}^{R}$ to update the fluid in cell $j+1$ (see Fig. 2).

\subsection{Level-Set Formulation}

We demonstrate how this algorithm is implemented in a level-set model. It is assumed that $(\rho, u, p, \psi)$ are available at each grid cell $j$ at the beginning of the calculation. We proceed as follows:

1. Use $\psi$ to determine the location of the material interface.

2. Compute the conserved variables $W=(\rho, \rho u E)$ in each cell, using the appropriate EOS as determined by $\psi$ in (9).

3. If $\psi_{j} \cdot \psi_{j+1}>0$ then (no interface) $\gamma_{l}=\gamma_{r} \equiv \gamma$. Compute one intercell flux $F_{j+\frac{1}{2}}\left(W_{j}\right.$, $\left.W_{j+1} ; \gamma\right)$ using the (common) value of $\gamma$ in the data.

4. Otherwise compute two intercell fluxes at $x_{j+\frac{1}{2}}, F_{j+\frac{1}{2}}^{L}\left(W_{j}, W_{j+1} ; \gamma_{l}\right)$ and $F_{j+\frac{1}{2}}^{R}$ $\left(W_{j}, W_{j+1} ; \gamma_{r}\right)$.

5. Use $F_{j+\frac{1}{2}}^{L}\left(W_{j}, W_{j+1} ; \gamma_{l}\right)$ to update $W_{L}=W_{j}^{n}$ and $F_{j+\frac{1}{2}}^{R}\left(W_{j}, W_{j+1} ; \gamma_{r}\right)$ to update $W_{R}=W_{j+1}^{n}$.

6. Compute the new primitive variables $U=(\rho, u, p)_{j}^{n+1}$, using frozen values of $\gamma$, namely $\gamma_{l, r}^{n}$.

7. Compute the level-set function $\psi_{j}^{n+1}$.

We make the following observations:

- The proposed algorithm is general and is independent of the method of discretization.

- The algorithm is obviously not conservative, since across interfaces the flux out of cell $j$ is different from the flux into cell $j+1$. However, the two-flux update affects only the total energy, while not affecting mass and momentum. (See Section 4.3 below.)

- At each given cell, the solution update from time level $n$ to $n+1$ "sees" only one fluid, with $\gamma=\gamma_{j}^{n}$. Contact surfaces are therefore oscillation-free, as is the case for single fluids. In particular, as shown in $(14,15)$ if $u$ and $p$ are uniform in the data, they will remain uniform in the solution. 
- Numerical diffusion across captured material interfaces does not cause spurious pressure oscillations to arise.

\subsection{Mass-Fraction Formulation}

The above algorithm may be implemented in a mass-fraction model in an almost identical fashion, simply by replacing the condition in Step 3 above by the condition (indicating no interface)

$$
\left|Y_{j+1}-Y_{j}\right|<\epsilon
$$

for some small value of $\epsilon$ (in [20] this condition was used to identify material fronts with $\epsilon=0.05)$.

An interesting variation is to use the two-flux formulas everywhere in the domain, yielding a scheme which appears to be nonconservative everywhere, but effectively is nonconservative only where $\gamma_{j} \neq \gamma_{j+1}$. An appealing advantage of the mass-fraction model, over a level-set formulation, is that it is capable of handling flows where the material front is not present initially but is "born" during the computation, for example due to chemical reaction (see Section 5).

\subsection{Conservation Error Analysis}

Two of the steps in the above algorithm are nonconservative. The first one, Step 5, is obvious since two adjacent cells are updated by different flux formulas. The second one is less obvious and lies in the combination of Steps 7 and 2. We first note that, depending on the EOS, a given cell pressure $p$ corresponds to different internal energy. Once the new conserved variables, $W_{j}^{n+1}$, have been computed, $p_{j}^{n+1}$ is recovered using the EOS with frozen thermodynamics. Only then is the level-set function updated, and with it the thermodynamics. If during the time step, the material interface has propagated through to the next cell, converting the pressure back to energy, using the EOS of "the other" fluid, then introduces energy conservation errors.

We show below that these two sources of conservation errors have opposing effects on the solution.

\subsubsection{Conservation Error Due to Different Flux Formulas}

The following analysis is for Roe's discretization method [29], which is used in the numerical examples section. Using $r_{k}, \lambda_{k}$, and $\alpha_{k}$ to denote the eigenvectors, eigenvalues, and wave strengths of the $k$ th characteristic field (see Section (2.1)), we write

$$
\begin{array}{lll}
r_{1}=(1, u-a, H-u a)^{T}, & \lambda_{1}=u-a, & \alpha_{1}=\frac{\Delta p-\rho a \Delta u}{2 a^{2}}, \\
r_{2}=\left(1, u, \frac{1}{2} u^{2}\right)^{T}, & \lambda_{2}=\tilde{u}, & \alpha_{2}=\frac{a^{2} \Delta \rho-\Delta p}{a^{2}}, \\
r_{3}=(1, u+a, H+u a)^{T}, & \lambda_{1}=u+a, & \alpha_{1}=\frac{\Delta p+\rho a \Delta u}{2 a^{2}} .
\end{array}
$$


Using $\lambda^{ \pm}$to indicate positive and negative eigenvalues, we have

$$
\begin{aligned}
& F_{j+\frac{1}{2}}^{L}\left(W_{L}, W_{R} ; \gamma_{L}\right)=F_{j}^{L}+\sum_{\lambda_{k}^{L-}} \alpha_{k}^{L} \lambda_{k}^{L} r_{k}^{L}, \\
& F_{j+\frac{1}{2}}^{R}\left(W_{L}, W_{R} ; \gamma_{R}\right)=F_{j+1}^{R}-\sum_{\lambda_{k}^{R+}} \alpha_{k}^{R} \lambda_{k}^{R} r_{k}^{R},
\end{aligned}
$$

and the flux difference is

$$
F_{j+\frac{1}{2}}^{R}-F_{j+\frac{1}{2}}^{L}=\left(F_{j+1}^{R}-F_{j}^{L}\right)-\left(\sum_{\lambda_{k}^{R+}} \alpha_{k}^{R} \lambda_{k}^{R} r_{k}^{R}+\sum_{\lambda_{k}^{L-}} \alpha_{k}^{L} \lambda_{k}^{L} r_{k}^{L}\right) .
$$

Clearly, if $\gamma_{L}=\gamma_{R}$ this expression vanishes. Across a material front, $\Delta u=\Delta p=0$ and the above expression reduces to

$$
\begin{aligned}
\left(F_{j+\frac{1}{2}}^{R}-F_{j+\frac{1}{2}}^{L}\right) & =\left(F_{j+1}^{R}-F_{j}^{L}\right)-u \Delta \rho\left(\begin{array}{c}
1 \\
u \\
\frac{1}{2} u^{2}
\end{array}\right) \\
& =\left(\begin{array}{c}
\rho u \\
\rho u^{2}+p \\
u(E+p)
\end{array}\right)^{R}-\left(\begin{array}{c}
\rho u \\
\rho u^{2}+p \\
u(E+p)
\end{array}\right)^{L}-u \Delta \rho\left(\begin{array}{c}
1 \\
u \\
\frac{1}{2} u^{2}
\end{array}\right) \\
& =\left(\begin{array}{c}
u \Delta \rho \\
u p \Delta\left(\frac{1}{\gamma-1}\right)+\frac{1}{2} u^{3} \Delta \rho
\end{array}\right)-u \Delta \rho\left(\begin{array}{c}
1 \\
u \\
\frac{1}{2} u^{2}
\end{array}\right) \\
& =\left(\begin{array}{c}
0 \\
0 \\
u p \Delta\left(\frac{1}{\gamma-1}\right)
\end{array}\right) .
\end{aligned}
$$

In $m$ time steps the accumulated conservation error is

$$
m \Delta t\left(\begin{array}{c}
0 \\
0 \\
u p \Delta\left(\frac{1}{\gamma-1}\right)
\end{array}\right)
$$

\subsubsection{Conservation Errors Due to "Unfreezing” of Thermodynamics}

Converting the primitive variables $(\rho, u, p)$ back to conserved variables, after "unfreezing" the thermodynamics, changes the internal energy and hence alters the total energy balance.

The total energy change is

$$
\Delta x\left(\left.E\right|_{\gamma=\gamma_{L}}-\left.E\right|_{\gamma=\gamma_{R}}\right)=\Delta x\left(\frac{p}{\gamma_{L}-1}-\frac{p}{\gamma_{R}-1}\right)=-\Delta x p \Delta\left(\frac{1}{\gamma-1}\right) .
$$


The number of time steps it takes for the interface to propagate through one grid cell is such that

$$
m u \Delta t / \Delta x \approx 1
$$

We observe that the two sources of error (26) and (27) have opposing effects on the solution, and from (28) they are nearly cancelling. The numerical examples in the next section display this kind of behavior. There is a build up of the conservation error due to the different flux formulas, which is then offset by the interface crossing to the next grid cell (see Fig. 8).

\section{NUMERICAL EXAMPLES}

The following examples illustrate the numerical difficulties encountered by naive discretization schemes of (1)-(3) described in Section 2 [1, 23, 26, 31] and demonstrate the ability of the single-fluid algorithm of Section 4 and the quasi-conservative scheme of Section 3.2.2 to handle these difficulties for problems involving ideal as well as stiff fluids. Conservation errors are computed, and convergence tests are included. We also present two examples where chemical reactions take place. They show that nonphysical oscillations of the kind described in this paper may be detrimental for reactive flow problems and that the single-fluid algorithm and the quasi-conservative method are capable of reliably solving such problems. Unless specified otherwise, the number of grid points is 200 and the CFL number is 0.8 .

\subsection{Test Case 1: Numerical Failures}

We solve (1)-(3a) using a second-order Roe-type scheme with superbee flux limiter [30]. The initial conditions correspond to the shock-tube data

$$
\begin{aligned}
& (\rho, u, p, \gamma)_{L}=(1,0,1,1.6) \\
& (\rho, u, p, \gamma)_{R}=(0.125,0,0.1,1.2)
\end{aligned}
$$

Figure 3 shows the computed velocity by the first-order Roe scheme applied to the massfraction model [1], the $\gamma$-model [31], and the level-set model [26]. The exact solution is also shown. All computations clearly suffer from large inaccuracies, with the velocity field clearly failing to maintain a uniform value across the material front.

\subsection{Test Case 2: Isolated Material Front}

We next consider an isolated material front and illustrate the behavior of the conservative scheme [1] and two nonconservative schemes: the single-fluid scheme and the quasiconservative scheme. Initial data are given by

$$
\begin{aligned}
& (\rho, u, p, \gamma)_{L}=(1,1,1,1.6), \\
& (\rho, u, p, \gamma)_{R}=(0.1,1,1,1.4) .
\end{aligned}
$$

Second-order calculations are shown in Fig. 4, which presents density, velocity, pressure, and $\gamma$ solution profiles, together with exact solutions. As expected, the single-fluid schemes 


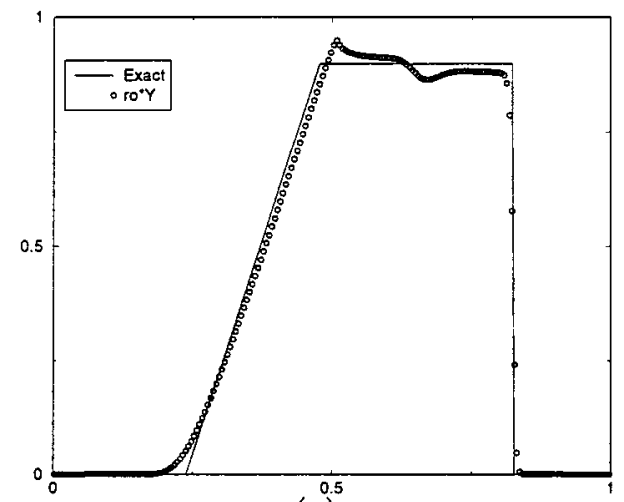

(a)

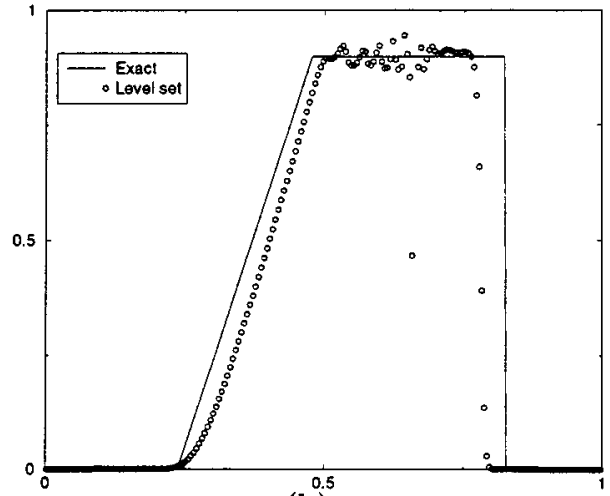

(b)

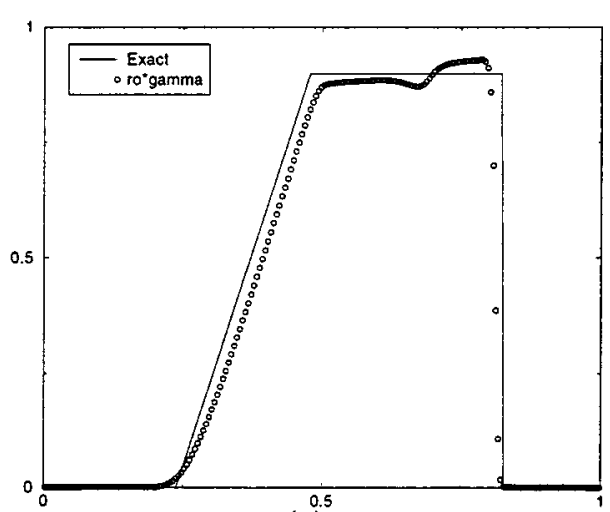

(c)

FIG. 3. Velocity by first-order schemes: (a) mass-fraction model, (b) level-set model, (c) $\gamma$-model.

(both mass-fraction and level-set formulations) and the quasi-conservative scheme maintain uniform velocity and pressure but the fully conservative scheme produces oscillatory solution. We note that these errors decay with mesh refinement, but they do so extremely slowly (see for example convergence tests in [2, 19]).

\subsection{Test Case 3: Two-Fluid Shock-Tube Problem}

In the next example, we consider the following shock-tube initial data:

$$
\begin{aligned}
& (\rho, u, p, \gamma)_{L}=(1,0,1,1.4) \\
& (\rho, u, p, \gamma)_{R}=(0.125,0,0.1,1.6)
\end{aligned}
$$

Figure 5 shows second order-computed solutions by the two single-fluid schemes (massfraction and level-set formulations) and by the quasi-conservative scheme. The results by all schemes are in very good agreement with the exact solution; in particular they are all oscillation-free and have the correct shock strength and speed. Results by the conservative scheme are not included, but as in the previous example, they are oscillatory near the contact, and while they eventually converge to zero with mesh refinement, they do so very slowly. Note also that increasing the order of accuracy of the scheme does not improve the quality of the results of the conservative scheme [2, 19]. 

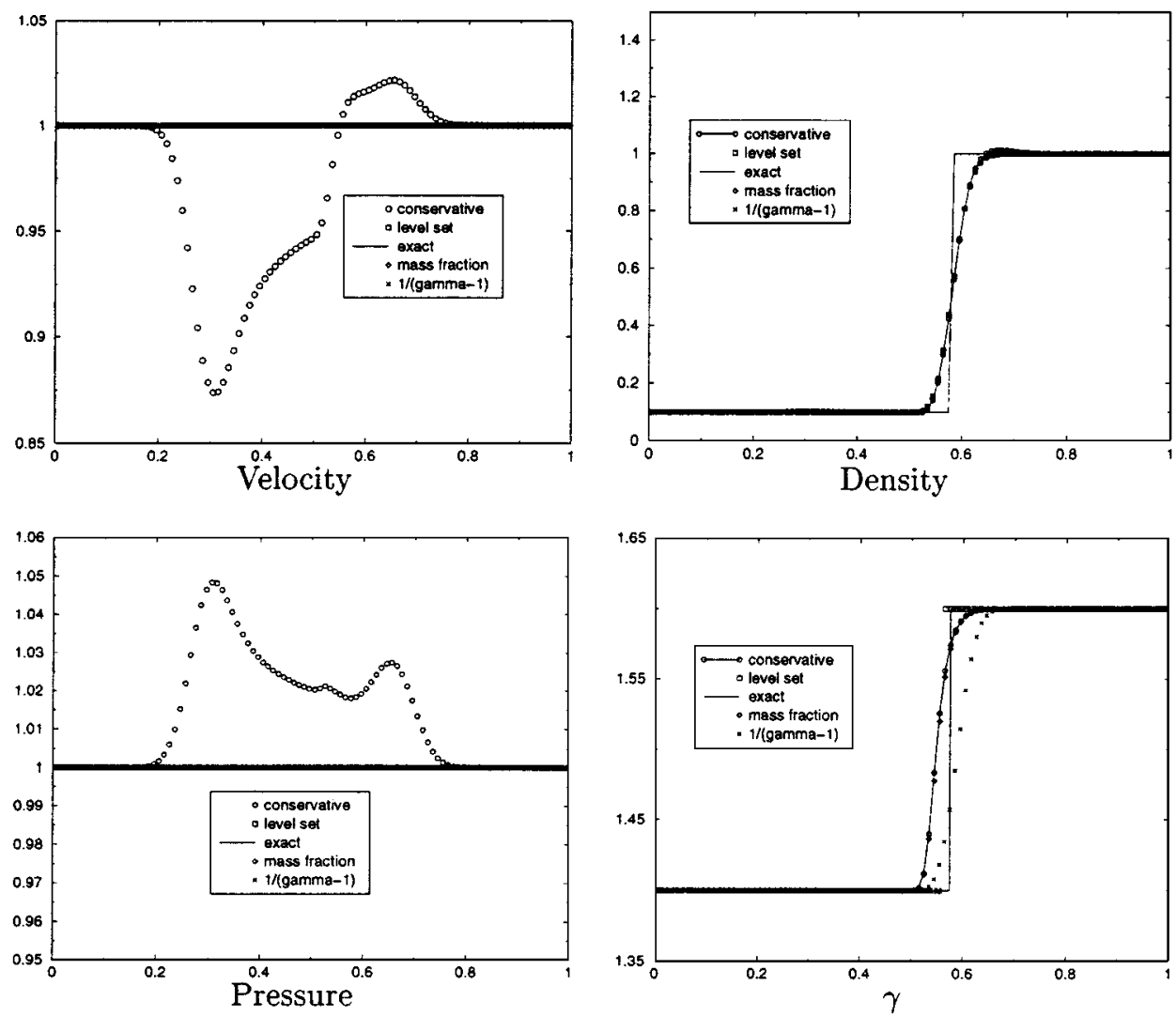

FIG. 4. Propagating material front computed by (i) the fully conservative scheme and by the single-fluid algorithm in (ii) mass-fraction, and in (iii) level-set formulation.

\subsection{Test Case 4: Two-Fluid Stiff Shock-Tube Problem}

The next example represents a more stiff shock-tube calculation. Initial data are given by

$$
\begin{aligned}
& (\rho, u, p, \gamma)_{L}=(1,0,0.2,1.4), \\
& (\rho, u, p, \gamma)_{R}=(1,0,500,1.6) .
\end{aligned}
$$

Solutions are again by the single-fluid scheme and by the quasi-conservative scheme. The results are shown in Fig. 6 for second-order calculations, with 800 grid points. The schemes clearly demonstrate their ability to compute strong shocks without deterioration in the quality of the results. In particular, we note that all computed solutions are oscillation-free and that, despite the fact that the single-fluid schemes are not conservative, results for this strong shock case are in excellent agreement with the exact solution.

We observe slight overshoots at the corner of the rarefaction fan and note that they are due to the stiffness of the problem, not the multifluid modelling. Indeed, similar behaviour can be observed in single fluid calculations.

\subsection{Test Case 5: Gas-Liquid Shock-Tube Problem}

We now consider a gas-liquid shock-tube test case using the stiffened equation of state (3b), taken from [32]. The initial conditions correspond to a severe water-air shock tube 

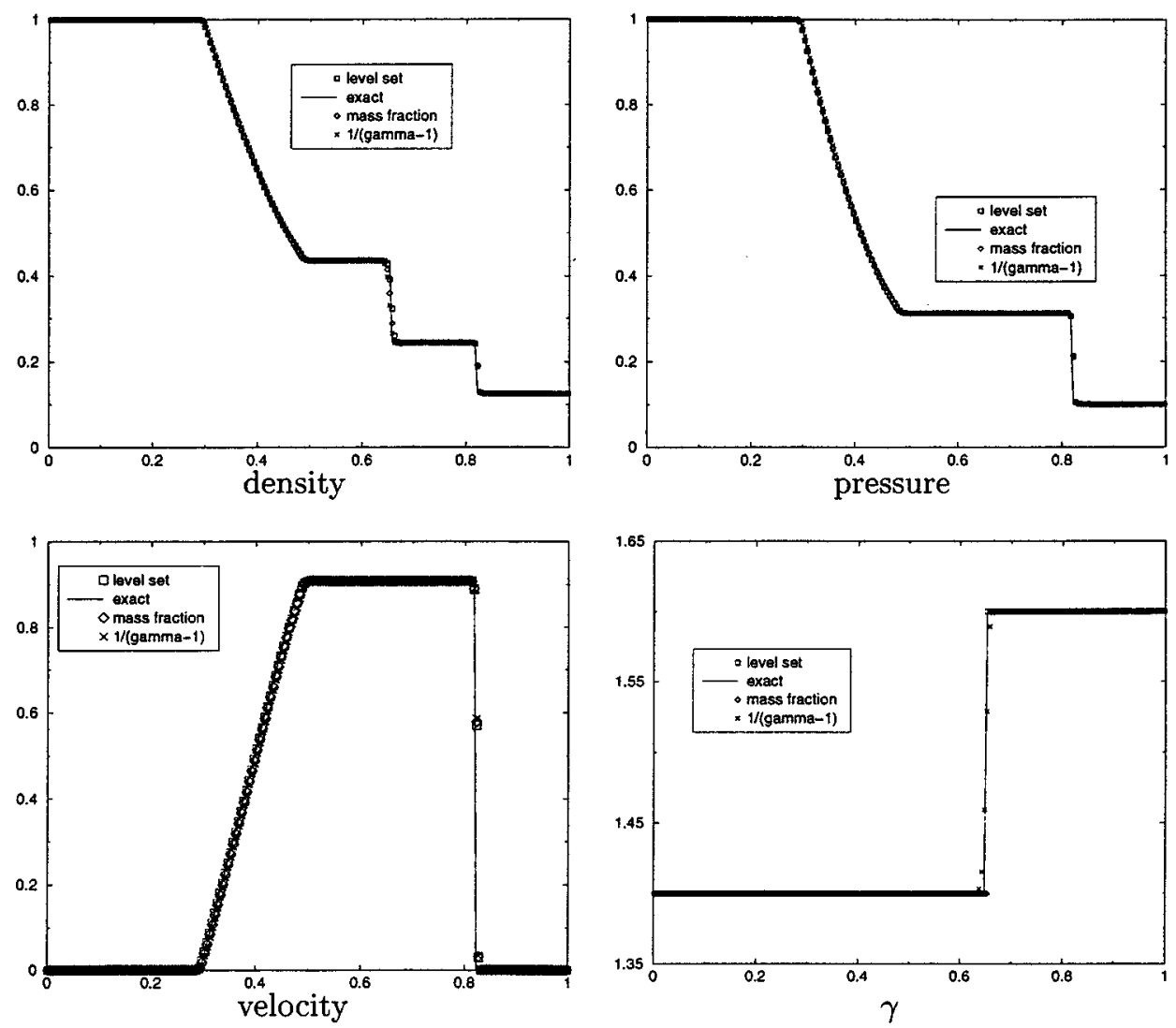

FIG. 5. Solutions for the shock-tube problem (31) by the single-fluid schemes and the quasi-conservative scheme.

given by

$$
\begin{aligned}
& \left(\rho, u, p, \gamma, p_{\infty}\right)_{L}=\left(1000,0,10^{9}, 4.4,6 \cdot 10^{8}\right), \\
& \left(\rho, u, p, \gamma, p_{\infty}\right)_{R}=\left(50,0,10^{5}, 1.4,0\right) .
\end{aligned}
$$

Results by the second-order single-fluid level-set scheme with minmod limiter are shown in Fig. 7. The agreement between the exact and numerical solutions is excellent, despite the severeness of the initial conditions and the fact that the method does not strictly conserve the total energy. Figure 8 shows the total energy and energy conservation errors as a function of time. The results are in agreement with the conservation error analysis of Section 4.3. The error exhibits fluctuations: It accumulates for a few steps before dropping as a result of the interface crossing mesh cell. More importantly, we note that even for this severe test case, the energy conservation error is extremely small, of the order of $0.2 \%$, and appears to reach saturation once the numerical solution has settled into a quasi-steady state. Convergence tests conducted in the next section indicate that these errors further decay to zero with mesh refinement, establishing that the method is essentially conservative. The same test problem was solved by the quasi-conservative scheme in [32], and the quality of the solution is similar. Finally, we note that because $p_{\infty}$ is very large, fully conservative 

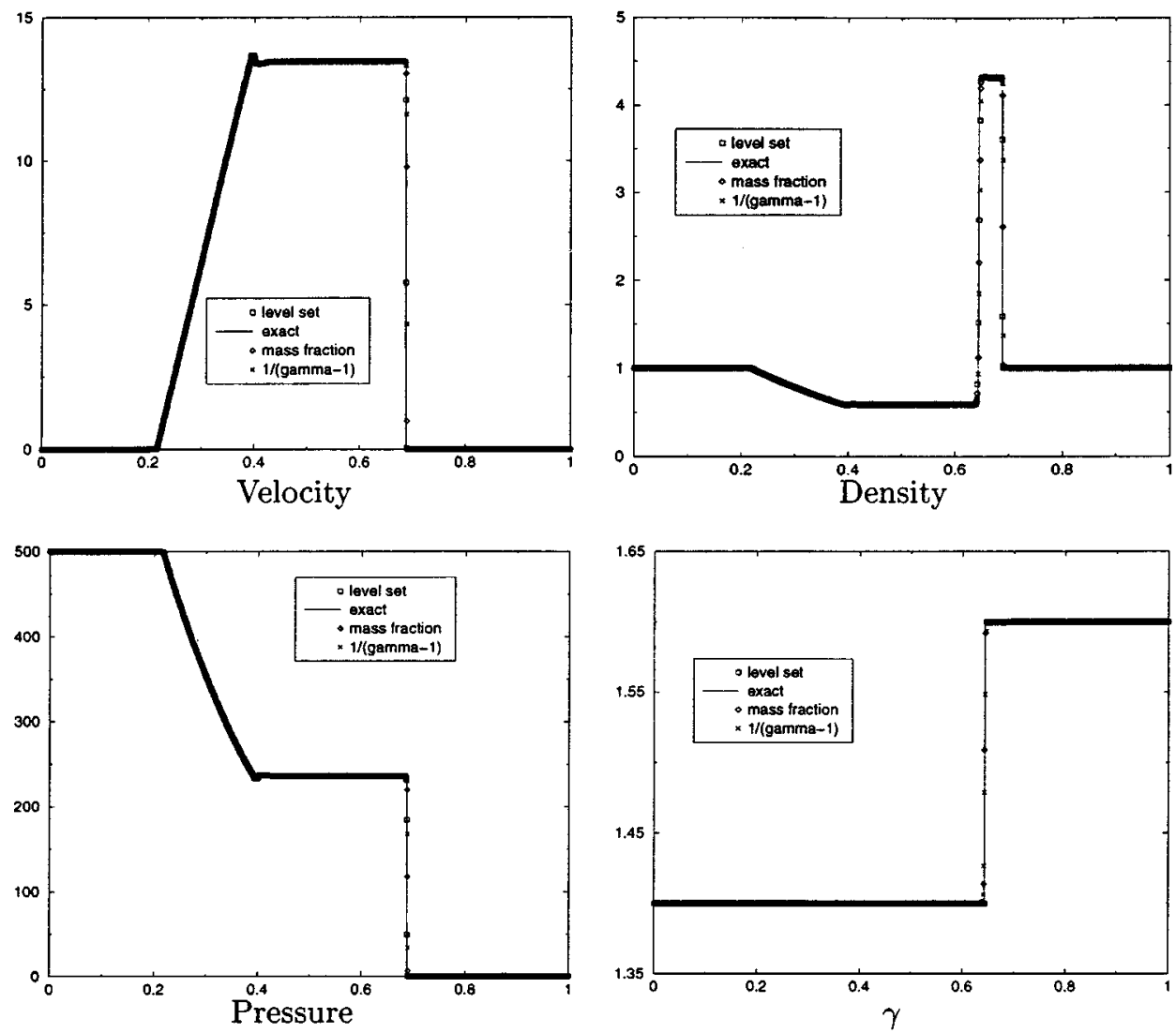

FIG. 6. Solutions for the stiff shock-tube problem (32) by the single-fluid schemes and the quasi-conservative scheme.

schemes have a hard time computing this test case, often failing because $p+p_{\infty}$ becomes negative.

\subsection{Convergence}

The test cases presented show excellent agreement between computed and exact solutions for a variety of problems involving moderate to strong shock waves. In particular, the shocks and material fronts appear to propagate with the correct speed and strength, and the rarefaction fans appear to expand at the correct rate. This is a strong indication that the overall loss of conservation does not affect the solution in a substantial way. In this section, we conduct mesh convergence studies for the various test cases. We compute relative conservation errors in total energy as a function of the number of grid points. The results are summarized in Tables II, III, and IV. Due to the fluctuating nature of the conservation error (see Fig. 8), we have measured the maximum error over the integration time period. The results all correspond to the same final time, so that finer grids imply that more time steps were taken.

In all cases, we see that the errors are extremely small (a fraction of a percent in general) and converge to zero (roughly like $\mathcal{O}(\Delta x)$ with mesh refinement. We further note that the 

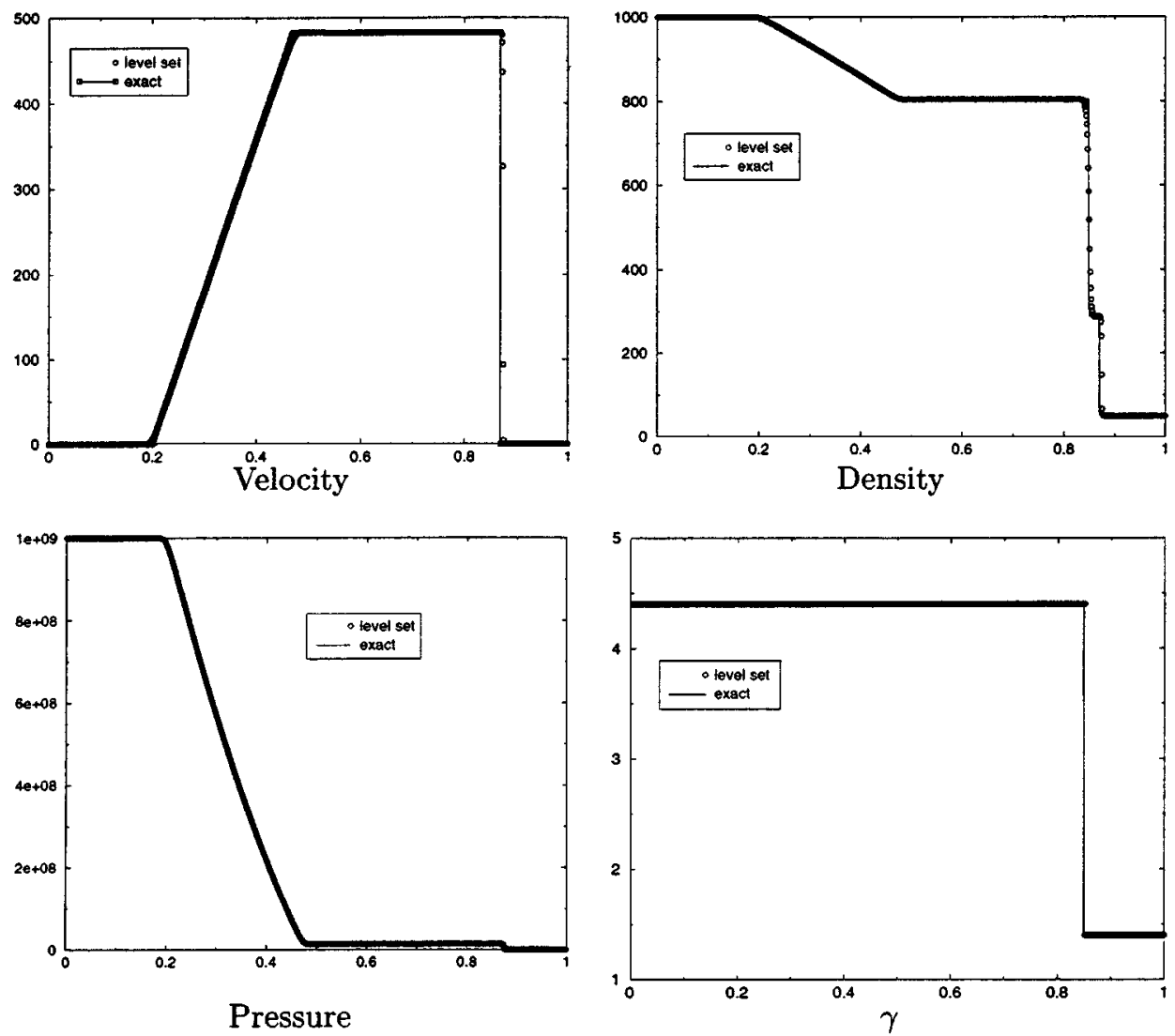

FIG. 7. Water-air shock-tube problem (33) by the level-set single-fluid scheme.

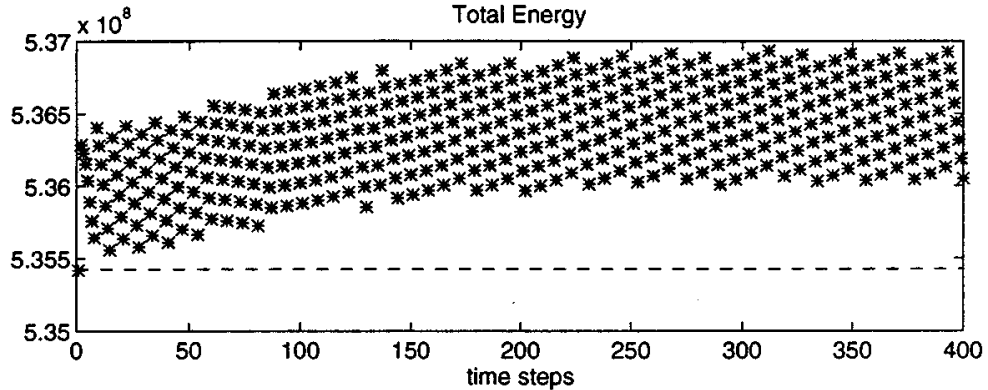

Total Energy Errors - \%

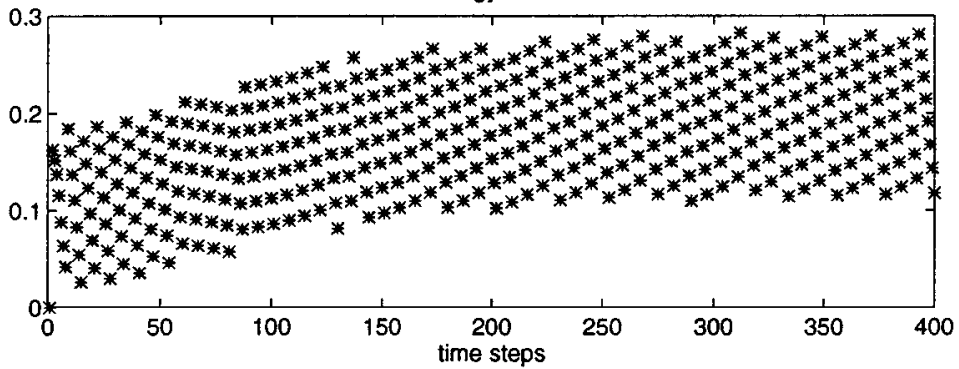

FIG. 8. Total energy (top) and relative conservation errors (bottom) for test case (33). 
TABLE II

Relative Error (in \%) in Total Energy at $\boldsymbol{t}=\mathbf{0 . 0 1}$ for Contact Discontinuity Test Case (30)

\begin{tabular}{cccccc}
\hline & \multicolumn{2}{c}{ Level-set } & & \multicolumn{2}{c}{ Mass-fraction } \\
\cline { 2 - 3 } \# of Points & 1st Order & 2nd Order & & 1st Order & 2nd Order \\
\hline 100 & 3.60 & 0.69 & & 5.6 & 2.1 \\
200 & 1.70 & 0.30 & & 3.22 & 1.11 \\
400 & 1.18 & 0.29 & & 2.26 & 0.63 \\
800 & 0.84 & 0.29 & & 1.59 & 0.35 \\
1600 & 0.58 & 0.22 & & 1.12 & 0.20 \\
3200 & 0.41 & 0.19 & & 0.79 & 0.11 \\
6400 & 0.29 & 0.13 & & 0.56 & 0.06 \\
\hline
\end{tabular}

TABLE III

Relative Error (in \%) in Total Energy at $\boldsymbol{t}=\mathbf{0 . 1 9}$ for Stiff

Shock-Tube Test Case (32)

\begin{tabular}{cccccc}
\hline & \multicolumn{2}{c}{ Level-set } & & \multicolumn{2}{c}{ Mass-fraction } \\
\cline { 2 - 3 } \cline { 5 - 6 } \# of Points & 1st Order & 2nd Order & & 1st Order & 2nd Order \\
\hline 100 & 2.08 & 0.72 & & 1.07 & 0.32 \\
200 & 1.43 & 0.36 & & 0.84 & 0.18 \\
400 & 1.06 & 0.18 & & 0.64 & 0.09 \\
800 & 0.80 & 0.09 & & 0.47 & 0.04 \\
1600 & 0.55 & 0.05 & & 0.34 & 0.02 \\
3200 & 0.41 & 0.03 & & 0.25 & 0.01 \\
6400 & 0.30 & 0.02 & & 0.18 & 0.005 \\
\hline
\end{tabular}

TABLE IV

Relative Error (in \%) in Total Energy at $\boldsymbol{t}=\mathbf{0 . 0 0 0 2}$ for Stiffened Shock-Tube Test Case (33)

\begin{tabular}{ccc}
\hline & \multicolumn{2}{c}{ Level-set } \\
\cline { 2 - 3 } \# of Points & 1st Order & 2nd Order \\
\hline 100 & 1.89 & 1.18 \\
200 & 0.97 & 0.70 \\
400 & 0.48 & 0.42 \\
800 & 0.24 & 0.24 \\
1600 & 0.18 & 0.18 \\
3200 & 0.11 & 0.06 \\
6400 & 0.09 & 0.03 \\
\hline
\end{tabular}


error and the convergence rate appear not to depend on shock strength or on the stiffness of the problem.

\subsection{Test Case 6: Chemistry}

A major weakness of level-set formulations is their inability to handle flows in which the material interfaces are not present initially, but rather get generated during wave interaction. This, for example, occurs in reactive flows. Consider the model specified by

$$
\begin{aligned}
\rho_{t}+(\rho u)_{x} & =0, \\
(\rho u)_{t}+\left(\rho u^{2}+p\right)_{x} & =0, \\
E_{t}+(u(E+p))_{x} & =0, \\
Y_{t}+u Y_{x} & =(1-Y) f(T),
\end{aligned}
$$

With $f$ given by

$$
f(T)= \begin{cases}0 & \text { if } T \leq T_{0}, \\ \exp \left(\frac{H_{0}}{T}\right) & \text { otherwise. }\end{cases}
$$

This model corresponds to the chemical reaction $\Sigma_{1} \rightarrow \Sigma_{2}$. Initial data are given by

$$
\begin{aligned}
& (\rho, u, p, \gamma)_{L}=(1,50,50,1.4), \\
& (\rho, u, p, \gamma)_{R}=(1,-50,50,1.4),
\end{aligned}
$$

with physical parameters $\gamma_{1}=1.4, \gamma_{2}=1.6, C_{v 1}=C_{v 2}=1, H_{0}=5000$, and $T_{0}=500$.

The problem is solved by the quasi-conservative scheme, which is modified as follows to take the chemistry into account: Given $W^{n}=\left(\rho^{n}, \rho^{n} u^{n}, E^{n}, \alpha^{n}\right)$,

1. Estimate $\alpha^{n+1 / 2}$ by

(a) computing $T^{n}=\frac{p^{n}}{\left(\gamma^{n}-1\right) \rho^{n}}$ (here we assume $C_{v 1}=C_{v 2}=1$ ),

(b) computing $Y^{n}$, i.e., inverting

$$
\frac{1}{\alpha^{n}}+1=\frac{Y^{n} C_{p 1}+(1-Y) C_{p 2}}{Y^{n} C_{v 1}+(1-Y) C_{v 2}}
$$

(c) computing

$$
Y^{n+1 / 2}= \begin{cases}Y^{n} & \text { if } \frac{p^{n}}{\rho^{n}} \leq 500 \\ 1-\left(1-Y^{n}\right) \exp \left(-\exp \left(\frac{H_{0}}{T^{n}}\right)\right) & \text { else }\end{cases}
$$

(d) computing $\alpha^{n+1 / 2}$ using (35).

2. Use the quasi conservative scheme with $W^{n+1 / 2}=\left(\rho^{n}, \rho^{n} u^{n}, E^{n}, \alpha^{n+1 / 2}\right)$ and go to 1 .

The results are shown in Fig. 9. The mesh size is $\Delta x=0.5 \cdot 10^{-2}$, we use 1000 time steps, and the CFL number is 0.1 . Initially, only species $\Sigma_{1}$ is present. The initial temperature is uniform, at $T=125$. Due to wave interaction, $T$ increases rapidly and once it exceeds $T=500$, a chemical reaction is triggered, and species $\Sigma_{2}$ is produced. We have run the problem also with the single-fluid scheme in mass-fraction formulation. The results are virtually undistinguishable. Such a computation is not possible with level-set models, as they require that the interface be present already in the initial data. 


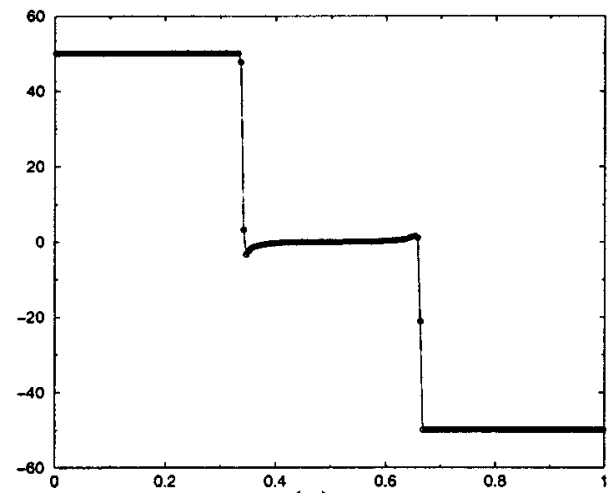

(a)

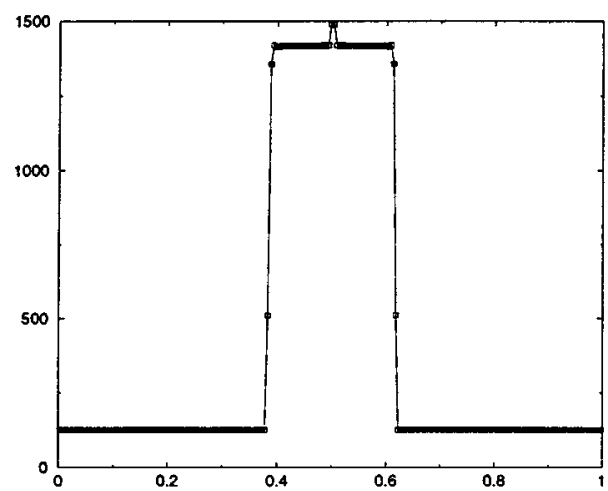

(c)

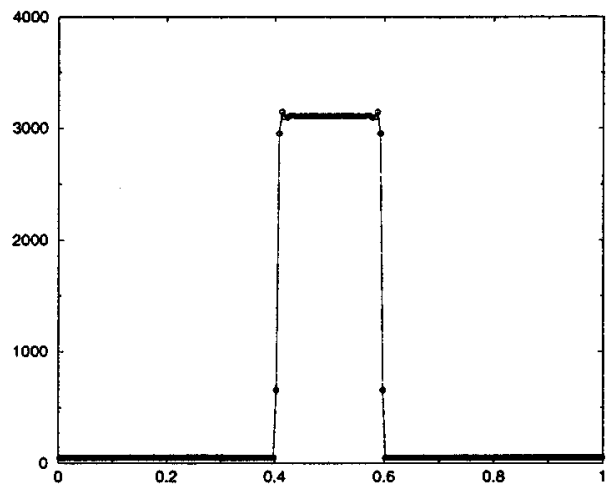

(b)

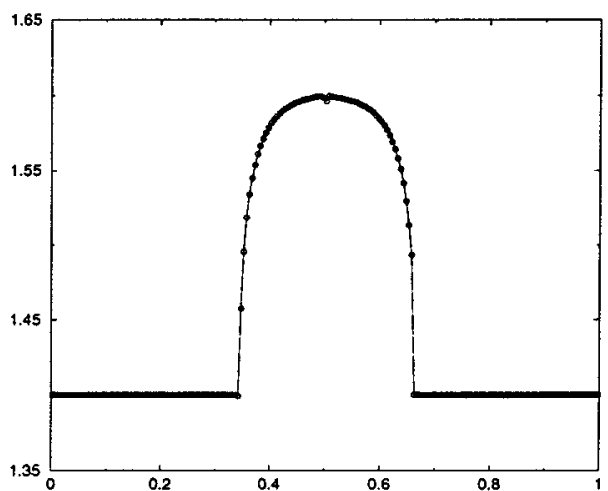

(d)

FIG. 9. (a) Velocity, (b) density, (c) temperature, and (d) $\gamma$ for test case (34).

\subsection{Test Case 7: Chemistry}

Finally, we show that computing oscillation-free material interfaces is crucial for obtaining reliable solutions in chemically reacting flows. Generating oscillations at a contact discontinuity may completely destroy the accuracy of a run. In this example, initial data are given by

$$
\begin{aligned}
& (\rho, u, p, \gamma)_{L}=(2.5,1,499,1.4), \\
& (\rho, u, p, \gamma)_{R}=(1.5,1,499,5 / 3),
\end{aligned}
$$

with physical parameters $\gamma_{1}=1.4, \gamma_{2}=5 / 3, C_{v 1}=C_{v 2}=1, H_{0}=7000$, and $T_{0}=500$. The initial conditions are set so that the temperature is uniform and $T<500$. Since the velocity and the pressure are also uniform, the solution is an inert material front propagating downstream at speed $u=1$, and no chemical reaction should occur.

Computations by the fully conservative scheme and the quasi-conservative scheme are shown in Fig. 10. The CFL number is $0.1, \Delta x=5.10^{-3}$, and we run 800 iterations.

As can be seen in Fig. 10, oscillations produced by the fully conservative scheme trigger a false chemical reaction, as $T$ exceeds the threshold value $T=500$. In comparison, the quasi-conservative scheme gives oscillation-free solutions, false chemical reaction is not 


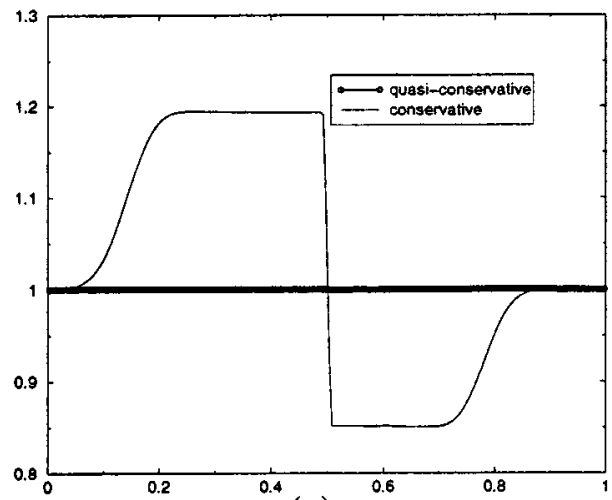

(a)

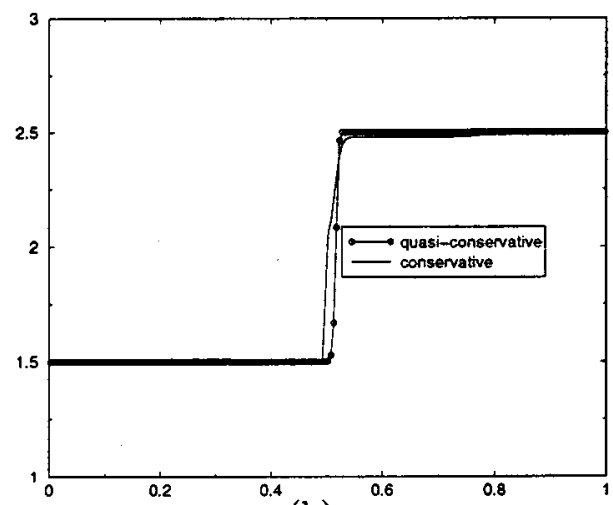

(b)

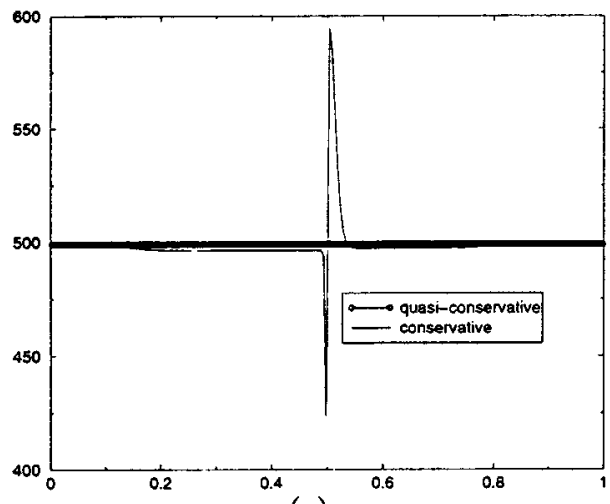

(c)

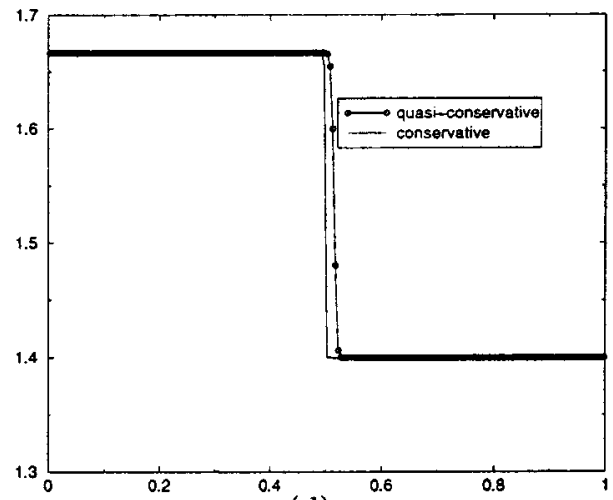

(d)

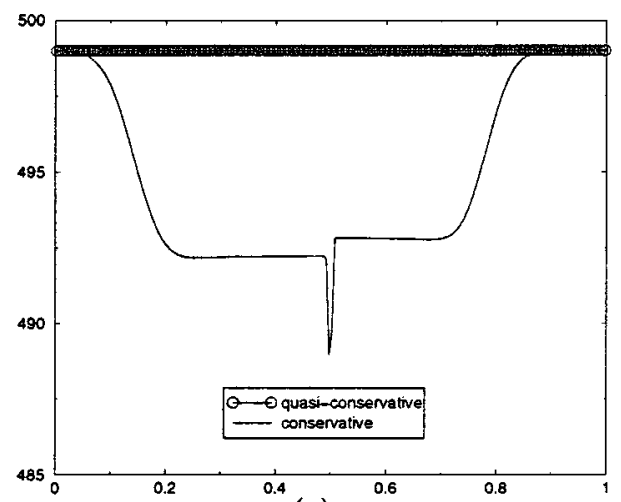

(e)

FIG. 10. (a) Velocity, (b) density, (c) temperature, (d) $\gamma$, and (e) pressure for test case (36).

triggered, and the inert material front propagates downstream in agreement with the exact solution.

\section{CONCLUDING REMARKS}

In this paper, we have surveyed a number of approaches to computing compressible multicomponent flows. While the eventual goal is to compute complex multimaterial flows 
in several space dimension, we have focused on the main modeling issues, discretization considerations, and guiding principles. Those are best illustrated and best understood in simple one-dimensional flow situations.

Ensuring the positivity of the mass fractions and ensuring the occurrence of pressure oscillations near material interfaces are typical of multimaterial flow simulations, the latter being a major obstacle in extending state-of-the-art schemes from single fluids to multifluids. These oscillations are entirely numerical artifacts, and we have reviewed several approaches to eliminate them, all involving various degrees of sacrificing strict conservation. The guiding principle is to ensure that if the flow has locally uniform velocity and pressure, the numerical scheme should be able to preserve it without generating disturbances. We noted that such oscillations are not present in single-fluid computations, and we introduced an extremely simple algorithm with a single-fluid flavor, based on two flux calculations across material fronts. The results, while not strictly conserving the total energy, exhibit conservation errors that are extremely small. These errors converge to zero with mesh refinement, and results are in excellent agreement with exact solutions even for severe test cases.

Finally, we note that if the flow is described by the balance equations for the individual species, rather than for the flow mixture, the same guiding principle can be used to develop algorithms that respect pressure equilibrium and at the same time do conserve mass of the individual species as well as momentum and energy of the flow mixture. This, however, comes at a price: The overall number of equations is roughly doubled in two-phase flows, and the system loses its underlying conservation form, as the result of momentum and energy exchange between the phases (although both are still conserved for the total mixture). The Volume-of Fluid algorithm [8, 27] essentially fits into this class of models. More recently, see [33] and also [9].

\section{REFERENCES}

1. R. Abgrall, Generalisation of the ROE scheme for the computation of mixture of perfect gases, Rech. Aérosp. 6, 1988.

2. R. Abgrall, How to prevent pressure oscillations in multicomponent flows: A quasi conservative approach, $J$. Comput. Phys. 125, 150 (1996).

3. R. Abgrall, B. N'Konga, and R. Saurel, Efficient numerical approximation of compressible multi-material flow for unstructured meshes, submitted for publication.

4. D. Chargy, R. Abgrall, L. Fezoui, and B. Larrouturou, Conservative numerical schemes for multicomponent inviscid flows, Rech. Aérosp. 2, 61 (1992).

5. I.-L. Chern, J. Glimm, O. McBryan, B. Plohr, and S. Yaniv, Front tracking for gas dynamics, J. Comput. Phys. 62, 83 (1986).

6. J. F. Clarke, S. Karni, J. J. Quirk, L. G. Simmonds, P. L. Roe, and E. F. Toro, Numerical computation of two-dimensional unsteady detonation waves in high energy solids, J. Comput. Phys. 106, 215 (1993).

7. J.-P. Cocchi and R. Saurel, A Riemann problem based method for the resolution of compressible material flows, J. Comput. Phys. 137, 265 (1997).

8. P. Colella, H. M. Glaz, and R. E. Ferguson, Multifluid algorithms for eulerian finite difference methods, unpublished, (1989).

9. F. Coquel, K. El-Almine, E. Godlewski, B. Perthame, and P. Rascle, A numerical method using upwind schemes for the resolution of two-phase flows, J. Comput. Phys. 136, 272 (1997).

10. R. Courant and K. O. Friedrichs, Supersonic Flow and Shock Waves, Pure and Applied Mathematics (Interscience Publishers, New York, 1948). 
11. S. F. Davis, An interface tracking method for hyperbolic systems of conservation laws, Appl. Numer. Math. 10, 447 (1992).

12. R. Fedkiw, T. Aslam, B. Merriman, and S. Osher, A non-oscillatory Eulerian approach to interfaces in multimaterial flows (the Ghost Fluid Method), J. Comput. Phys. 152, 457 (1999).

13. R. Fedkiw, T. Aslam, and S. Xu, The ghost fluid method for deflagration and detonation discontinuities, J. Comput. Phys. 154, 393 (1999).

14. R. Fedkiw, A. Marquina, and B. Merriman, An isobaric fix for the overheating problem in multimaterial compressible flows, J. Comput. Phys. 148, 545 (1999).

15. S. K. Godunov, A difference scheme for numerical computation of discontinous solutions of equations of fluids dynamics, Math. Sb. 47, 271 (1959).

16. M. J. Ivings, D. M. Causon, and E. F. Toro, On hybrid high resolution upwind methods for multicomponent flows, Z. Angew. Math. Mech. 77(9), 645 (1997).

17. M. J. Ivings, D. M. Causon, and E. F. Toro, On Riemann solvers for compressible liquids, Int. J. Numer. Meth. Fluids 3, 395 (1998).

18. P. Jenny, B. Mueller, and H. Thomann, Correction of conservative Euler solvers for gas mixtures, J. Comput. Phys. 132, 91 (1997).

19. S. Karni, Multi-component flow calculations by a consistent primitive algorithm, J. Comput. Phys. 112, 31 (1994).

20. S. Karni, Hybrid multifluid algorithms, SIAM J. Sci. Comput. 17, 1019 (1996).

21. S. Karni, Compressible bubbles with surface tension, in 16th International Conference on Numerical Methods in Fluid Dynamics, edited by Ch.-H. Bruneaux (Springer-Verlag, Berlin/New York, 1998), pp. 506512.

22. S. Karni, A level-set scheme for compressible interfaces, in Numerical Methods for Wave Propagation, edited by E. F. Toro and J. F. Clarke (Kluwer Academic Publishers, Dordrecht, 1988), pp. 253-274.

23. B. Larouturou, How to preserve the mass fraction positive when computing compressible multi-component flows, J. Comput. Phys. 95, 59 (1991).

24. B. Larouturou and L. Fezoui, On the Equations of Multicomponent Perfect or Real Gas Inviscid Flow, in Lecture Notes in Mathematics (Springer-Verlag, Berlin/New York, 1989), Vol. 1402, pp. 69-97.

25. G. H. Miller and E. G. Puckett, A high order Godunov method for multiple condensed phases, J. Comput. Phys. 128, 134 (1996).

26. W. Mulder, S. Osher, and J. Sethian, Computing interface motion: The compressible Rayleigh-Taylor and Kelvin-Helmholtz instabilities, J. Comput. Phys. 100, 209 (1992).

27. W. F. Noh and P. R. Woodward, Slic (simple line interface calculation), in Springer Lecture Notes in Physics (Springer-Verlag, Berlin/New York, 1976), Vol. 59, pp. 330-339.

28. J. J. Quirk and S. Karni, On the dynamics of a shock-bubble interaction, J. Fluid Mech. 318, 129 (1996).

29. P. L. Roe, Approximate riemann solvers, parameter vectors and difference schemes, J. Comput. Phys. 43, 357 (1981).

30. P. L. Roe, Fluctuations and signals-A framework for numerical evolution problems, in Numerical Methods for Fluid Dynamics, edited by K. W. Morton and M. J. Baines (Academic Press, New York, 1982), pp. 219257.

31. P. L. Roe, A New Approach to Computing Discontinuous Flows of Several Ideal Gases, Technical Report (Cranfield Institute of Technology, 1984).

32. R. Saurel and R. Abgrall, A simple method for compressible multifluid flows, SIAM J. Sci. Comp. 21(3), (1999).

33. R. Saurel and R. Abgrall, Some models and methods for compressible multifluid and multiphase flows, J. Comput. Phys. 150, 425 (1999).

34. K.-M. Shyue, An efficient shock-capturing algorithm for compressible multicomponent problems, J. Comput. Phys. 1, 208 (1998).

35. K.-M. Shyue, A fluid-mixture type algorithm for compressible multicomponent flow with Van der Waals equation of state, J. Comput. Phys. 1, 43 (1999). 
36. V. Ton, Improved shock-capturing methods for multicomponet and reacting flows, J. Comput. Phys. 128, 237 (1996).

37. E. F. Toro, Primitive, conservative and adaptive schemes for hyperbolic conservation laws, in Numerical Methods for Wave Propagation (Manchester 95), edited by E. F. Toro and J. F. Clarke (Kluwer Academic Publishers, Dordrecht, 1998), pp. 253-274.

38. B. van Leer, Towards the ultimate conservative difference scheme: 2 . Monotonicity and conservation combined in a second-order scheme, J. Comput. Phys. 14, 361 (1974).

39. K. Xu, BGK-based scheme for multicomponent flow calculations, J. Comput. Phys. 1, 122 (1998). 